\title{
SÍNTESIS Y CARACTERIZACIÓN DE GRAFENO MULTICAPA MAGNETIZADO
}

\author{
Mercedes Puca Pacheco*a, María Guadalupe Neira Velázquezb
}

\begin{abstract}
RESUMEN
La presente investigación tuvo como objetivo la síntesis y caracterización de grafeno multicapa magnetizado y para ello se propuso un diseño factorial $2^{2}$ con un punto central, para evaluar el efecto del tamaño de las nanopartículas de grafito y la relación másica de magnetita/grafeno sobre la magnetización. El grafeno multicapa magnetizado se obtuvo en dos etapas, en la primera se sonificó el grafito con dodecilsulfato de sodio (SDS) en medio acuoso y en la segunda etapa se realizó la coprecipitación in situ de la magnetita, logrando la formación de las nanoláminas de grafeno multicapa. Mediante el análisis por espectroscopia Raman se encontró la formación de grafeno multicapa. Mediante los resultados de la difracción de rayos $\mathrm{X}$ se verificó la fase cristalina de la magnetita y el grado de cristalinidad. Mediante análisis morfológico, utilizando el microscopio de barrido (SEM), se confirmaron las características microestructurales de estos materiales. En conclusión, la magnetización del material compuesto de grafeno y magnetita no depende de la relación másica de magnetita/ grafeno porque el grafeno fue magnetizado y tampoco depende de los tamaños de partículas del grafito sonicado y grafeno multicapa magnetizado.

Palabras claves: Magnetita, grafeno multicapa, magnetización, cristalinidad, grafito.
\end{abstract}

\section{SYNTHESIS AND CHARACTERIZATION OF MAGNETIZED MULTILAYER GRAPHENE}

\begin{abstract}
The present research aimed at the synthesis and characterization of magnetized multilayer graphene and for this, a $2^{2}$ factorial design with a central point was proposed, to evaluate the effect of the size of the graphite nanoparticles and the magnetite / graphene mass ratio on magnetization. The magnetized multilayer graphene was obtained in two stages, in the first the graphite was sonified with sodium dodecyl sulfate (SDS) in aqueous medium and in the second stage the magnetite in situ coprecipitation was carried out, achieving the formation

a Facultad de Química e Ingeniería Química, Universidad Nacional Mayor de San Marcos UNMSM. Av. Germán Amézaga N³75, Lima 01, CP 15081, Perú. email: mpucap@gmail.com

b Centro de Investigación en Química Aplicada, Blvd. Enrique Reyna Hermosillo, No. 140, Col. San José de los Cerritos, CP. 25294, Saltillo, Coahuila, México.
\end{abstract}


of multilayer graphene. By means of the analysis by Raman spectroscopy the formation of multilayer graphene was found. By means of the X-ray diffraction results, the crystalline phase of the magnetite and the degree of crystallinity were verified. By morphological analysis using the scanning microscope (SEM), the microstructural characteristics of these materials were confirmed. In conclusion, the magnetization of the composite material of graphene and magnetite does not depend on the mass ratio of magnetite / graphene because the graphene was magnetized and neither does it depend on the particle sizes of the sonified graphite and magnetized multilayer graphene.

Key words: Magnetite, multilayer graphene, magnetization, crystallinity, graphite.

\section{INTRODUCCIÓN}

En la actualidad, el grafeno ocupa un lugar importante y está permitiendo abrir nuevas vías al desarrollo de materiales funcionales por su obtención a partir de un material barato como el grafito. El grafeno es una nanoestructura de carbono bidimensional (2D), con hibridación $\mathrm{sp}^{2}$, que ha sido de gran interés en las comunidades de física, química y materiales en todo el mundo desde que fue descubierto por Geim y Novoselov ${ }^{1}$, quienes usaron cinta adhesiva "scotch" para despegar láminas de grafeno a partir del grafito. El interés por el grafeno se debe a sus excelentes propiedades tales como: alta conductividad térmica ${ }^{2}$, la resistencia mecánica comparable a la de los nanotubos de carbono que lo han convertido en material de gran interés de estudio en muchas investigaciones del siglo $\mathrm{XXI}^{3}$, con un módulo de Young de 1 Terapascal, es 100 veces más resistente que el acero y 6 veces más ligero que el mismo y mejora las propiedades térmicas y ópticas ${ }^{3}$, las buenas propiedades de transporte ${ }^{4}$ y su considerable efecto termoeléctrico ${ }^{4}$, la cual hace que el grafeno sea una gran promesa para su uso en diversos materiales de almacenamiento de energía ${ }^{5}$, sistemas de suministro de fármacos ${ }^{6}$, biosensores ${ }^{7}$, compuestos poliméricos ${ }^{8}$, supercondensadores ${ }^{9}$, nanoelectrónica ${ }^{10}$ y en otras áreas.

Los métodos de exfoliación oxidativa pueden producir potencialmente grandes cantidades de óxido de grafeno (GO), una nanocapa similar al grafeno que generalmente es defectuosa y requiere más tratamientos para reducirlo a óxido de grafeno reducido $(\mathrm{RGO})^{11}$. Sin embargo, las aplicaciones del grafeno siguen siendo limitadas debido a su alto costo y su baja capacidad de producción actual. Por ello, actualmente, la exfoliación de grafito es un enfoque prometedor para la producción a gran escala de grafeno. La exfoliación en fase líquida, mediante el empleo de disolventes orgánicos ${ }^{12,13}$ o surfactantes ${ }^{14}$, es una alternativa a la exfoliación mecánica que se basa en aumentar la distancia interlaminar entre las capas de grafito para disminuir las fuerzas de interacción (Van der Waals) existentes entre ellas y poder separar las láminas ${ }^{15}$.

En la presente investigación, se empleó la sonicación de una mezcla de grafito, tensioactivo (SDS) y agua, para la obtención de grafeno multicapa, y luego la precipitación de magnetita en la dispersión acuosa de grafeno multicapa. Una posible aplicación del nanocompuesto obtenido es en la limpieza de aguas contaminadas o aguas de mar por metales pesados, mediante remoción magnética, cuyo estudio se realizará en un futuro trabajo de investigación. 


\section{PARTE EXPERIMENTAL}

\section{Reactivos y materiales}

Los reactivos utilizados en este trabajo fueron: dodecilsulfato de sodio (SDS) con pureza $>$ $98,5 \%$, cloruro de hierro (II) $\left(\mathrm{FeCl}_{2} \cdot 4 \mathrm{H}_{2} \mathrm{O}\right)$, cloruro de hierro (III) $\left(\mathrm{FeCl}_{3} \cdot 6 \mathrm{H}_{2} \mathrm{O}\right)$, hidróxido de amonio al $30 \% \mathrm{~m} / \mathrm{m}$ y ácido clorhídrico al $37 \% \mathrm{~m} / \mathrm{m}$, suministrados por Merck Peruana. Para la síntesis del grafeno se empleó grafito de Sigma-Aldrich. Se empleó agua destilada, desionizada y filtrada. El nitrógeno fue de grado UAP de Infra, purificado adicionalmente al pasarlo por una columna Oxiclear.

\section{Caracterización}

La estructura cristalográfica y la presencia de la magnetita fueron analizadas por difracción de Rayos-X (DRX) empleando un difractó $\neg$ metro marca BRUKER, modelo D8-FOCUS. Generador de Rayos-X con voltaje de salida del tubo $=40 \mathrm{kV}$ y corriente de salida del tubo $=40 \mathrm{~mA}$ en un rango de medición de 10 a $80^{\circ} \mathrm{C}$ en la escala de $2 \theta$.

Las mediciones del tamaño de partícula se realizaron por dispersión de luz (DLS) en un equipo modelo Malvern Zetasizer Nano-ZS90 a $25^{\circ} \mathrm{C}$.

Las propiedades magnéticas de las nano $\neg$ partículas fueron determinadas usando el Magnetómetro de Muestra Vibrante (MMV) modelo 6000 de Quantum Design, con un campo aplicado entre $-20,0$ a 20,0 kOe a temperatura ambiente.

Se obtuvieron los espectros de las ondas correspondientes al grafeno con el espectrofotómetro Raman Horiba One Xplora, utilizando un láser de $785 \mathrm{~nm}$ con una potencia de $50 \mathrm{~mW}$, de 0 a $3500 \mathrm{~cm}^{-1}$.

Para el estudio morfológico se empleó un Microscopio Electrónico de Barrido (MEB) XL20 de Phillips, en donde las muestras fueron analizadas con un voltaje entre 6,5 y $15 \mathrm{kV}$ y magnificaciones entre 1000 y $10000 \mathrm{x}$.

\section{Diseño experimental}

Se planteó un diseño $2^{2}$ con un punto central, siendo las variables independientes el tiempo de dispersión del grafito y relación másica de magnetita/grafeno (RMG) (Ver tabla 1) 
Tabla 1. Diseño factorial $2^{2}$ con un punto central.

\begin{tabular}{cccc}
\hline Código & $\begin{array}{c}\text { Diseño factorial } \\
{[\text { Tiempo(h)] }[\mathrm{RMG}]}\end{array}$ & $\begin{array}{c}\text { Tiempo de } \\
\text { sonicación del } \\
\text { grafito } \\
(\mathrm{h})\end{array}$ & $\begin{array}{c}\text { Relación másica } \\
\text { Magnetita/Grafeno } \\
(\mathrm{RMG}) \\
(\%)\end{array}$ \\
\hline MA & $(-)(-)$ & 5 & $40 / 60$ \\
MB & $(-)(+)$ & 5 & $60 / 40$ \\
MC & $(0)(0)$ & 10 & $50 / 50$ \\
MD & $(+)(-)$ & 15 & $40 / 60$ \\
ME & $(+)(+)$ & 15 & $60 / 40$ \\
\hline
\end{tabular}

\section{Obtención del grafito con diferentes tamaños de partícula}

Para ello se llevó a cabo la dispersión de una mezcla compuesta por $5 \mathrm{~g}$ de grafito; $0,5 \mathrm{~g}$ de dodecilsulfato de sodio (SDS) y $300 \mathrm{~mL}$ de agua destilada, mediante el empleo de un baño de ultrasonido modelo S30H "ELMA"" ALEMAN", por un tiempo de 5 horas, a una temperatura constante de $40{ }^{\circ} \mathrm{C}$. Se realizaron éstas mezclas, de la misma manera, para otros tiempos de dispersión de 10 y 15 horas. (ver tabla 2).

Tabla 2. Muestras de grafito dispersado por ultrasonido.

\begin{tabular}{lc}
\hline Código & $\begin{array}{r}\text { Tiempo de agitación por } \\
\text { ultrasonido (h) }\end{array}$ \\
\hline MF & 5 \\
MG & 10 \\
MH & 15 \\
\hline
\end{tabular}

\section{Síntesis de la magnetita sobre grafito dispersado por ultrasonido}

Una vez obtenidas las tres mezclas dispersadas de grafito MF, MG y MH obtenidas a diferentes tiempos, estas se vertieron por separado en balones de tres bocas de $500 \mathrm{ml}$; se calentaron hasta $70{ }^{\circ} \mathrm{C}$, en ambiente inerte con nitrógeno gaseoso, se mantuvieron en agitación a una velocidad de $500 \mathrm{rpm}$ y una vez alcanzada una temperatura constante de 70 ${ }^{\circ} \mathrm{C}$, se adicionaron las cantidades necesarias de solución de $\mathrm{FeCl} 3$ al $24 \%$ m/v y FeCl2 al 12 $\% \mathrm{~m} / \mathrm{v}$ (para obtener proporciones de magnetita y grafito de $40 / 60,60 / 40$ y $50 / 50$ ) y luego de 5 minutos se dosificó la cantidad necesaria de $\mathrm{NH} 4 \mathrm{OH}$ concentrado a un flujo volumétrico de $1 \mathrm{ml} / \mathrm{min}$ empleando una bomba de jeringa modelo New Era NE-550 y una vez finalizada la alimentación del $\mathrm{NH} 4 \mathrm{OH}$, se dejó reaccionar por 1 hora adicional.

Una vez finalizada, se decantó el grafito o el grafeno multicapa magnetizado, luego se eliminó el líquido sobrenadante, después se filtró y se realizó el proceso de lavado del grafito 
o grafeno multicapa magnetizado con agua bidestilada hasta obtener un $\mathrm{pH}$ de 7 en el líquido de filtrado. Finalmente, se colocó el papel de filtro con el grafito o grafeno magnetizado en una placa Petri y se llevó a secar en una estufa de secado a $50{ }^{\circ} \mathrm{C}$ durante 5 días.

La síntesis de las nanopartículas de magnetita (NPM) por el método de precipitación, fue llevada a cabo a partir de una solución formada por cloruro de hierro (II) y cloruro de hierro (III) en presencia de hidróxido de amonio con la siguiente relación estequiométrica:

$9 \mathrm{FeCl}_{3 \text { (ac) }}+6 \mathrm{FeCl}_{2 \text { (ac) }}+39 \mathrm{NH}_{4} \mathrm{OH}_{\mathrm{cc}} \rightarrow 5 \mathrm{Fe}_{3} \mathrm{O}_{4(\mathrm{~s})}+39 \mathrm{NH}_{4} \mathrm{Cl}_{\text {(ac) }}+19 \mathrm{H}_{2} \mathrm{O}+1 / 2 \mathrm{H}_{2}$

\section{RESULTADOS Y DISCUSIÓN}

\section{Determinación del tamaño de partícula del grafito por DLS}

Se obtuvieron tamaños de partículas entre 206,7 y 243,9 nm, los cuales son mostrados en la tabla 3, y también podemos afirmar que a mayor tiempo de dispersión por ultrasonido se logra disminuir el tamaño de partícula (Ver figura 1).

Tabla 3. Diámetros de partícula del grafito por DLS.

\begin{tabular}{ccc}
\hline CÓDIGO & $\begin{array}{c}\text { Tiempo de agitación por } \\
\text { ultrasonido } \\
\text { (h) }\end{array}$ & $\begin{array}{c}\text { Diámetro de } \\
\text { partícula (nm) }\end{array}$ \\
\hline MF & 5 & 243,9 \\
MG & 10 & 226,9 \\
MH & 15 & 206,7 \\
\hline
\end{tabular}

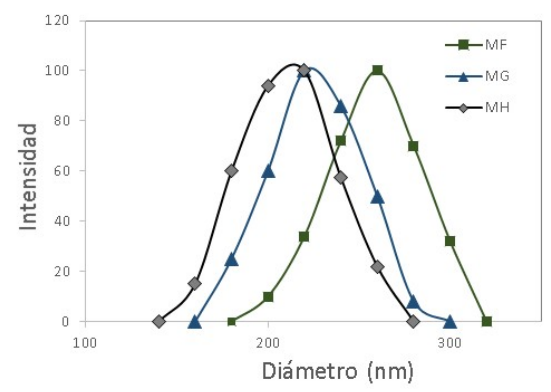

Figura 1. Distribución del tamaño de las partículas de grafito por DLS.

\section{Análisis por difracción de rayos $\mathrm{X}$ de muestras de grafito magnetizado}

En la figura 2, se muestran los difractogramas de las muestras: MA, MB, MC y MD en las cuales se formó la magnetita, mientras que en la muestra ME hubo formación de magnetita y goethita. Asimismo, se muestra que el grafito sonificado a 5, 10 y $15 \mathrm{~h}$, las cuales corresponden a las muestras MF, MG y MH respectivamente, se observan solo Grafito H: Sistema Hexagonal y Grafito R: Sistema Romboédrico. 
Mediante el difractograma de rayos X, se evidencia la formación de la magnetita cristalina por la presencia de los picos de difracción intensos indexados a (2 20 0), (3 11 1), (4 0 0 0), (4 2 2), (5 11 1) y (4 4 0) planos que aparecen en $2 \theta=30,15 ; 36,27 ; 43,32 ; 53,89 ; 57,13$ y 62,29 ${ }^{\circ}$, respectivamente, son consistentes con los datos XRD estándar para la fase cúbica $\mathrm{Fe} 3 \mathrm{O} 4$ con una estructura cúbica centrada en las caras (fcc).

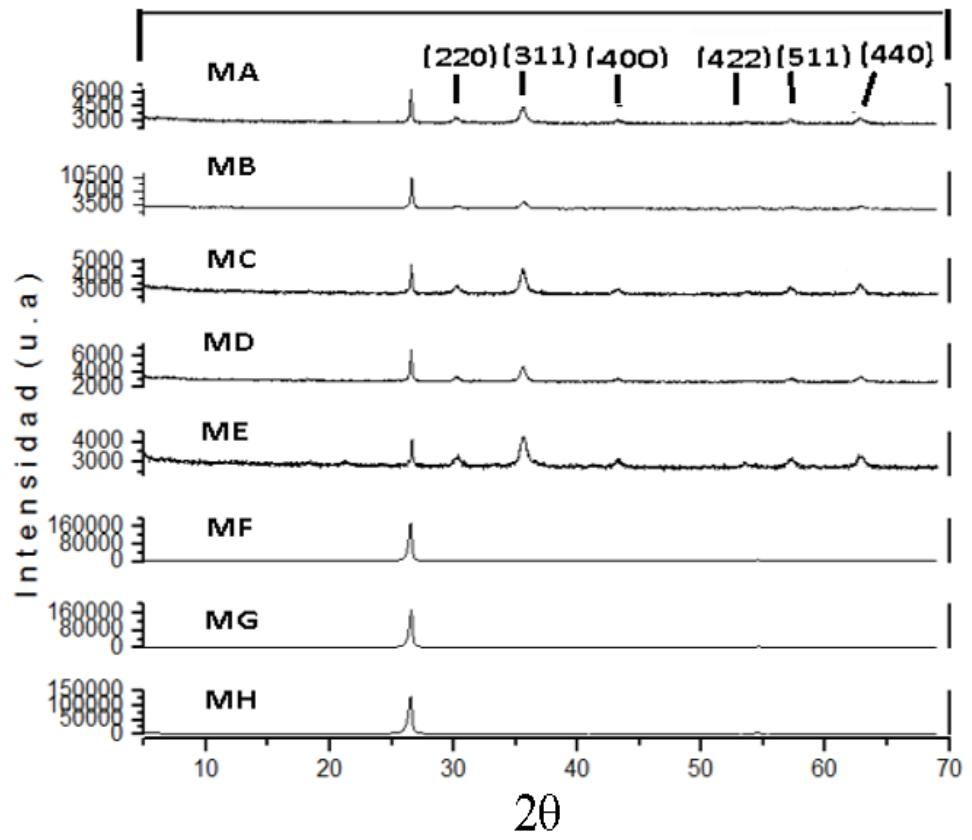

Figura 2. Comparación de difractogramas de rayos $\mathrm{X}$ de los nanocompuestos (MA, MB, MC, MD y ME) y grafito (MF, MG y MH).

El diámetro de la magnetita se calculó a partir de los difractogramas de rayos X, mediante la anchura a media altura (FWHM) del pico de reflexión del plano cristalino (311), siendo el pico más intenso de las reflexiones del perfil de DRX y se empleó la fórmula de Scherrer16 la cual viene dada por la ecuación 1 .

$$
D=\frac{k \cdot \lambda}{\beta \cdot \cos \theta}
$$

Donde $\mathrm{K}$ es una función de la forma de partículas, en este caso se consideró un valor de 0.9 , $\lambda$ es la longitud de onda del haz de rayos X, $\beta$ es el ancho del pico a la altura media (FWHM) en radianes y $\theta$ el ángulo de Bragg en radianes.

A continuación, se muestra la tabla 4 con los diámetros de magnetita obtenidos en las muestras MA, MB, MC, MD y ME. 
Tabla 4. Diámetro de partículas de magnetita.

\begin{tabular}{cc}
\hline Muestra & Tamaño de grano $(\mathrm{nm})$ \\
\hline MA & 12,46 \\
MB & 12,13 \\
MC & 11,74 \\
MD & 15,69 \\
ME & 11,22
\end{tabular}

\section{Análisis por espectroscopia Raman}

En la figura 3, se muestra la comparación del espectro Raman del grafeno y del grafito medidos a 514,5 nm según Ferrari (2007)17. Se aprecian dos criterios de identificación: 1) Analizando las componentes del pico 2D, o 2) Analizando la razón de intensidades entre los picos G y 2D.
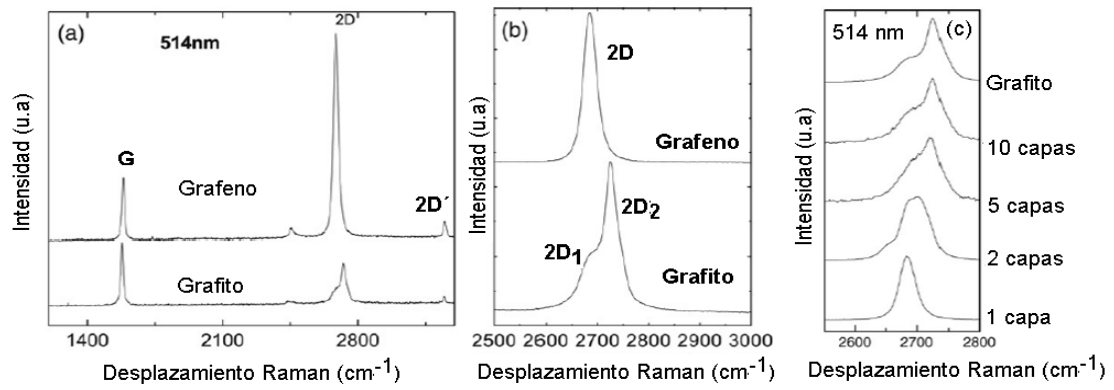

Figura 3. Espectro Raman del grafeno y grafito: (a) Con los picos G y 2D; (b) grafeno y grafito con los picos 2D para grafeno y 2D1 y 2D2 para grafito y (c) grafito y diferentes capas.

En la figura 4 se aprecian las bandas características del grafito, la imagen insertada muestra la forma del pico 2D típico de grafito en masa. La banda D es bastante pequeña, por lo que se deduce un alto orden estructural.

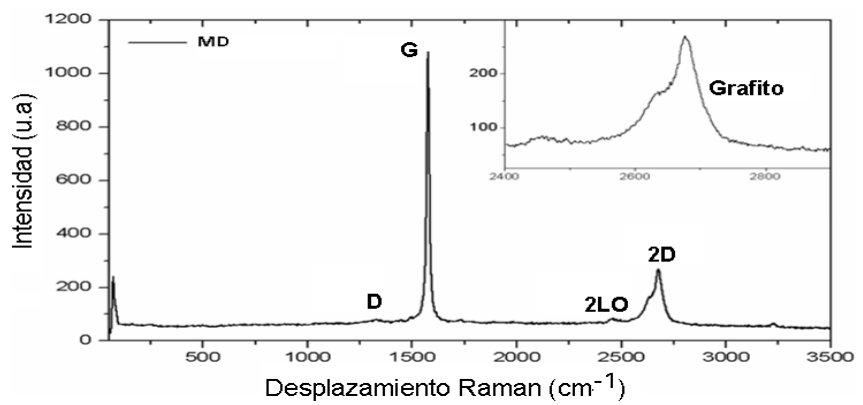

Figura 4. Espectro Raman de las nanopartículas de grafito-magnetita ( $\%$ Magnetita $=60 \%)$, con un tiempo de agitación por ultrasonido de $15 \mathrm{~h}$. 
En la figura 5, se aprecia el espectro Raman de la muestra MC con un $40 \%$ de magnetita, la cual se diferencia de la muestra MD, que en la imagen insertada se distingue que la forma del pico 2D refleja, aproximadamente, 10 capas de carbono. Además, se logra observar que la banda $\mathrm{D}$ tiene una mayor intensidad, corroborando la presencia de estados de bordes.

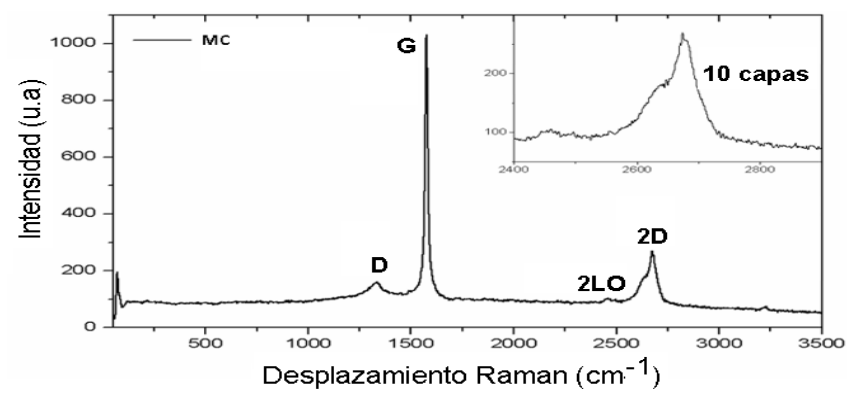

Figura 5. Espectro Raman de las nanopartículas de grafito-magnetita (\%Magnetita $=40 \%)$ con un tiempo de agitación por ultrasonido de $15 \mathrm{~h}$.

En la figura 6, se muestra el espectro de la muestra ME, en la cual se observan los picos del grafito y en la imagen insertada se observa que el pico 2D muestra una ligera asimetría que corresponde a aproximadamente 5 capas de carbono.

Por otro lado, en la figura 6, se observan picos pequeños (alrededor de $200 \mathrm{~cm}-1$ ) que corresponden al óxido de hierro. La línea de fondo (background) ascendente denota una alta fluorescencia a consecuencia del mal enfoque en la superficie de medida, lo que se apoya en la premisa que se trata de pocas capas de grafeno sobre una nanopartícula de óxido de hierro (Magnetita).

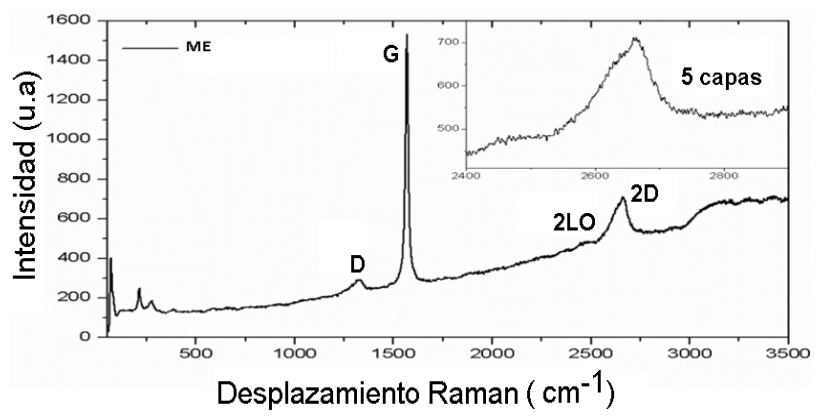

Figura 6. Espectro Raman de las nanopartículas de grafito-magnetita $(\mathrm{RM}=50 / 50)$ con un tiempo de agitación por ultrasonido de $10 \mathrm{~h}$. 
En la figura 7, se muestran los espectros Raman para las muestras MA y MB, en la cual coinciden en casi todos los picos, salvo los indicados con flechas que corresponden al grafito, como se evidenció en las gráficas previas. Hacer un análisis cuidadoso del espectro en morado (el que presenta grafito) es imposible debido a la superposición de picos que dificultan poder determinar el número de capas, pero a simple vista se trataría de grafito en masa.

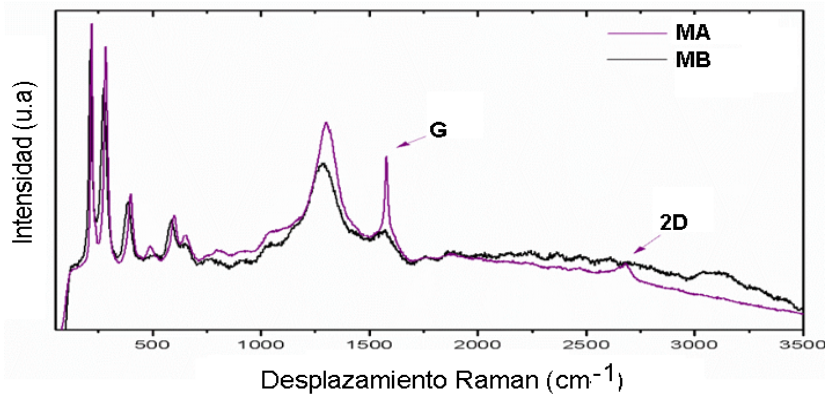

Figura 7. Espectro Raman de las nanopartículas de grafito-magnetita $(\mathrm{RM}=60 / 40$ y $\mathrm{RM}=$ 40/60) con un tiempo de agitación por ultrasonido de $5 \mathrm{~h}$.

\section{Análisis de magnetización}

En la tabla 5 y figura 8, se muestran los resultados de las magnetizaciones de las muestras, encontrándose una alta magnetización con un valor de 52,21 emu/g en promedio, cuando el grafito se agitó por un tiempo de 10 horas con un $50 \%$ de magnetita. Por otro lado, las propiedades magnéticas de este tipo de ferrita dependen fuertemente del tamaño de partícula. Según estudios realizados por Kumar et al., 2015, encontraron que cuando las partículas de $\mathrm{Fe}_{3} \mathrm{O}_{4}$ se reducen a escala nanométrica, sus propiedades magnéticas decaen: la magnetización de saturación $(M s)$, la remanencia magnética $(B r)$ y la fuerza coercitiva $(H c)$ disminuyen drásticamente $y$, en consecuencia, las nanopartículas exhiben un comportamiento denominado superparamagnético ${ }^{18}$. Por tanto, en esta investigación, se obtuvieron tamaños entre 11,22 y $15,69 \mathrm{~nm}$, dando valores de magnetización entre 38,15 y 52,41 Emu/g, como se muestra en la tabla 5 y figura 8 (a), (b) y (d), y comparado con lo obtenido en el artículo reportado por Puca et al., 2013 se reportaron magnetizaciones de un valor 31,82 emu/g para un diámetro de partícula promedio de $9 \mathrm{~nm}^{19}$. 
Tabla 5. Magnetización del nanocompuesto de grafeno multicapa-magnetita.

\begin{tabular}{cccccc}
\hline Código & $\begin{array}{c}\text { Diseño factorial } \\
\text { [tiempo(h)][RMG }]\end{array}$ & $\begin{array}{c}\text { Tiempo } \\
\text { de } \\
\text { sonificación } \\
\text { del grafito } \\
\text { (h) }\end{array}$ & $\begin{array}{c}\text { Relación másica } \\
\text { Magnetita/Grafeno } \\
(\mathrm{RMG})\end{array}$ & $\begin{array}{c}\text { Diámetro de } \\
\text { magnetita (nm) }\end{array}$ & $\begin{array}{c}\text { Magnetización } \\
\text { Emu/g }\end{array}$ \\
\hline MA & $(-)(-)$ & 5 & $40 / 60$ & 12,46 & 39,47 \\
MB & $(-)(+)$ & 5 & $60 / 40$ & 12,13 & 38,15 \\
ME & $(+)(+)$ & 15 & $60 / 40$ & 11,22 & 46,79 \\
MD & $(+)(-)$ & 15 & $40 / 60$ & 15,69 & 38,99 \\
MC & $(0)(0)$ & 10 & $50 / 50$ & 11,74 & 52,41 \\
MC & $(0)(0)$ & 10 & $50 / 50$ & 11,74 & 52,00 \\
\hline
\end{tabular}

Según el trabajo reportado por Peña-Rodriguez et al., 2018 evidenciaron un incremento lineal de la magnetización de saturación y del momento magnético en función de la cantidad de magnetita adicionada a la matriz polimérica ${ }^{20}$. Sin embargo, en el presente trabajo de investigación no se obtuvo un incremento de la magnetización al aumentar la concentración de magnetita en la relación másica de magnetita/grafeno y esto se atribuye a que el grafeno se ha magnetizado, es decir que, al formarse la magnetita, este le ha dotado al grafeno de magnetización. Así, el resultado fue una nanolámina imantada basada en grafeno. Por tanto, el material obtenido abre la posibilidad de que el grafeno tenga propiedades magnéticas.

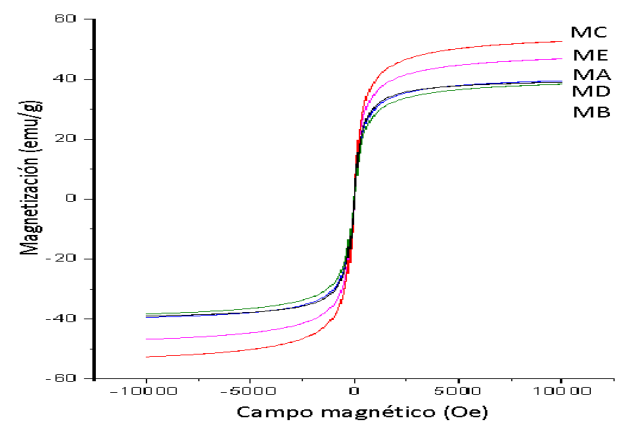

Figura 8. Espectro Raman de las nanopartículas de grafito-magnetita $(\mathrm{RM}=50 / 50)$ con un tiempo de agitación por ultrasonido de $10 \mathrm{~h}$. 
En la figura 9, se muestran las imágenes tomadas en el Microscopio Electrónico de Barrido (MEB) con un voltaje de $15 \mathrm{kV}$ y a diferentes magnificaciones entre 1000x y 10224x, de la superficie de las partículas de grafito y/o grafeno multicapa magnetizado, en donde se aprecian partículas de magnetita con morfología esferoidal en todos los casos, como lo observado en el trabajo reportado por Puca et al., $(2013)^{19}$, por lo que se demuestra que las partículas de magnetita han sido estabilizadas por la presencia del tensoactivo SDS, y que la presencia del grafito durante la reacción de precipitación con $\mathrm{FeCl}_{3}$ y $\mathrm{FeCl}_{2}$ en medio básico con $\mathrm{NH}_{4} \mathrm{OH}$, no ha afectado la morfología de la magnetita. También se aprecia, en las imágenes, la formación de plaquetas de grafito y nanoescamas de grafeno multicapa intercaladas en la matriz de magnetita, como se observó en el trabajo reportado por Sun et al., (2019) ${ }^{21}$. Las nanoláminas de grafeno se muestran en la imagen de $\mathrm{MB}$ y ME, aunque las láminas de grafeno observadas, no son muy delgadas, posiblemente por la formación de multicapas, así también se aprecian entre estas láminas a las nanopartículas de magnetita de forma esferoidal.

En la Figura 9, se observan en todas las muestras las aglomeraciones entre partículas que han sido atribuidas a interacciones magneto-dipolo entre las mismas, debido a que las láminas de grafeno multicapas están rodeados por las cargas introducidas por las moléculas remanentes del tensioactivo.
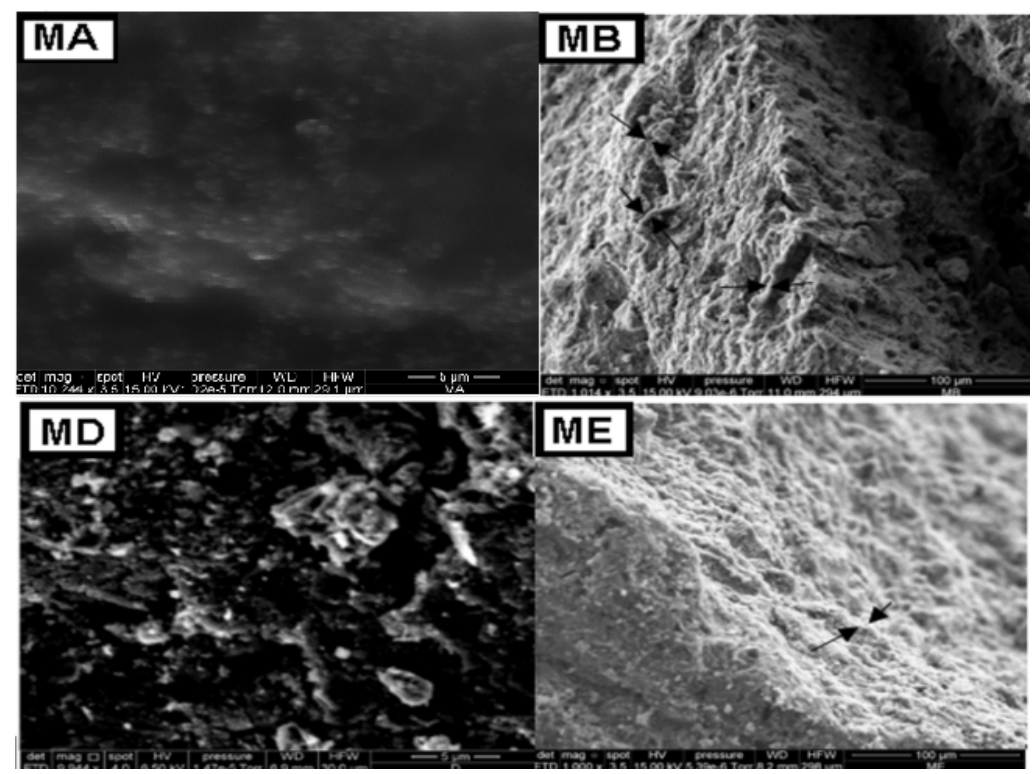

Figura 9. Imágenes obtenidas en el SEM, de las partículas de grafeno multicapa magnetizado: a) muestra MA; b) muestra MB; c) muestra MD y d) muestra ME 
En la tabla 6, se muestran los resultados de magnetización y diámetro de las partículas de magnetita obtenidas de acuerdo al diseño factorial 22 con un punto central, teniendo en cuenta las variables independientes: Diámetro de partícula del grafito y la relación másica de magnetita/grafeno (RMG).

Tabla 6. Magnetización del nanocompuestos de grafeno multicapa-magnetita.

\begin{tabular}{cccccc}
\hline Código & $\begin{array}{c}\text { Diseño factorial } \\
{[\mathrm{Dp}(\mathrm{nm})][\mathrm{RMG}]}\end{array}$ & $\begin{array}{c}\text { Dp grafito } \\
\text { Ultrasonido } \\
(\mathrm{nm})\end{array}$ & $\begin{array}{c}\text { DRM }] \\
\text { magnetita } \\
(\mathrm{nm})\end{array}$ & $\begin{array}{c}\text { Magnetización } \\
\text { Emu/g }\end{array}$ \\
\hline MA & $(+)(-)$ & 243,6 & $40 / 60$ & 12,46 & 39,47 \\
MB & $(+)(+)$ & 243,6 & $60 / 40$ & 12,13 & 38,15 \\
ME & $(-)(+)$ & 206,7 & $60 / 40$ & 11,22 & 46,79 \\
MD & $(-)(-)$ & 206,7 & $40 / 60$ & 15,69 & 38,99 \\
MC & $(0)(0)$ & 226,9 & $50 / 50$ & 11,74 & 52,41 \\
MC & $(0)(0)$ & 226,0 & $50 / 50$ & 11,75 & 52,00 \\
\hline
\end{tabular}

Se realizó la prueba de Shapiro-Wilk (prueba de normalidad) para los resultados de magnetización y diámetro de partícula de la magnetita y se obtuvieron valores de p-valor = 0,097 y 0,496 , respectivamente, y siendo en ambos casos valores mayores que $\alpha=0,05$. Por lo tanto, cumplen la prueba de normalidad.

La tabla 7, muestra el análisis de varianza para el diámetro de partícula de la magnetita y se aprecia que hay efecto significativo de la relación másica de magnetita/grafeno (RMG) ( $\mathrm{p}$-valor $=0,002)$, así como del diámetro de partícula del grafito ( $\mathrm{p}$-valor $=0,004)$ y existe interacción entre las variables independientes $(\mathrm{p}$-valor $=0,002)$ sobre el diámetro de partícula de la magnética, ya que sus p-valores son menores que $\alpha=0,05$, a un nivel de confianza del $95 \%$.

También, en la tabla 7, se muestra el análisis de varianza para la magnetización y se aprecia que no hay efecto del diámetro de partícula del grafito $(\mathrm{p}$ - valor $=0,045)$, ni de la relación másica de magnetita/grafeno ( $\mathrm{p}$-valor $=0,057$ ), ya que sus $\mathrm{p}$-valores son mayores que $\alpha=$ 0,05 , a un nivel de confianza del $95 \%$. Sin embargo, existe interacción de ambos factores (diámetro del grafito y relación másica de magnetita/grafeno) sobre la magnetización, ya que el valor-P es menor que $\alpha=0,05$ 
Tabla 7. Análisis de varianza para el diámetro de magnetita y la magnetización

\begin{tabular}{ll}
\hline Diámetro de magnetita $(\mathbf{n m})$ : & \\
Efectos principales & \\
A: Dp grafito ultrasonido(nm) & 0,004 \\
B: RMG & 0,002 \\
AB & 0,002 \\
Magnetización Emu/g: & \\
Efectos principales & \\
A: Dp grafito ultrasonido (nm) & 0,045 \\
B: RMG & 0,057 \\
AB & 0,040 \\
*Prueba estadística. &
\end{tabular}

\section{CONCLUSIONES}

El grafeno presenta extraordinarias propiedades mecánicas y electrónicas, pero no magnéticas. Sin embargo, la síntesis de la magnetita por coprecipitación in situ en la dispersión de grafito sonificado, permitió la separación de las láminas de grafito, para obtener nanoláminas de grafeno dotado de propiedades magnéticas.

Mediante el análisis estadístico se comprobó que, al variar la relación másica de grafeno/ magnetita en el nanocompuesto, no afecta la magnetización, lográndose obtener un valor máximo de 52,21 emu/g cuando la relación másica de grafeno y magnetita es de 1:1 y ello se debe a que mediante el proceso de obtención se le ha dotado al grafeno con propiedades magnéticas.

Los nanocompuestos de grafeno/magnetita sintetizados fueron estables y fueron sintetizados a partir de láminas de grafeno 3D obtenido por sonificación, dando la posibilidad de ser escalados a nivel industrial, ya que es un proceso barato y se evita la contaminación de grafeno por los reactivos empleados.

\section{REFERENCIAS}

1. Geim A, Kim P. Carbon Wonderland. Sci Am. 2008; 298(4):90-97.

2. Balandin A, Ghosh S, Bao W, Calizo, Teweldebrhan D, Miao F, Lau C.N. Superior Thermal Conductivity of Single-Layer Graphene. Nano Lett. 2008; 8: 902-907.

3. Lonkar SP, Deshmukh YS, Abdala AA. Recent advances in chemical modifications of grapheme. Nano Res. 2015; 8: 1039-1074.

4. Geim AK. Graphene: status and prospects. Science. 2009; 324: 1530-1534.

5. Zhang J, Zhao F, Zhang ZP, Chen N, Qu LT. Dimension-tailored functional graphene structures for energy conversion and storage. Nanoscale. 2013; 5: 3112-3126. 
6. Liu JQ, Cui L, Losic D. Graphene and graphene oxide as a new nanocarriers for drug delivery applications. Acta Biomater. 2013; 9: 9243-9257.

7. Kuila T, Bose S, Khanra P, Mishra AK, Kim NH, Lee JH. Recent advances in graphenebased biosensors. Biosens Bioelectron. 2011; 26: 4637-4648.

8. Du JH, Cheng HM. The fabrication, properties, and uses of graphene/polymer composites. Macromol. Chem Phys. 2012; 213: 1060-1077.

9. Iwan A, Chuchmala A. Perspectives of applied graphene: polymer solar cells. Prog Polym Sci. 2012; 37: 1805-1828.

10. Maiti UN, Lim J, Lee KE, Lee WJ, Kim SO. Three-dimensional shape engineered, interfacial gelation of reduced graphene oxide for high rate, large capacity supercapacitors. Adv Mater. 2014; 26: 615-619.

11. Wang C, Han X, Xu P, Zhang X, Du Y, Hu S, et al. The electromagnetic property of chemically reduced graphene oxide and its application as microwave absorbing material. Appl Phys Lett. 2011; 98:072906. doi: 10.1063/1.3555436

12. Paton KR, Varrla E, Backes C, Smith RJ, Khan U, O'Neill A, et al. Scalable production of large quantities of defect-free few-layer graphene by shear exfoliation in liquids. Nat Mater. 2014;13(6):624-630.

13. Bourlinos AB, Georgakilas V, Zboril R, Steriotis TA, Stubos AK. Liquid-phase exfoliation of graphite towards solubilized graphenes. Small. 2009; 5: 1841-1845.

14. Hernandez Y, Nicolosi V, Lotya M, Blighe FM, Sun Z, De S, et al. High-yield production of graphene by liquid-phase exfoliation of graphite. Nat Nanotechnol. 2008; 3: 563-568.

15. De S, King PJ, Lotya M, O'Neill A, Doherty EM, Hernandez Y, et al. Flexible, Transparent, Conducting Films of Randomly Stacked Graphene from Surfactant-Stabilized, OxideFree Graphene Dispersions. Small. 2010; 6(3), 458-464.

16. Cullity B. D. Elements of X-ray Diffraction. London: Prentice-Hall International; 2000.

17. Ferrari AC. Raman spectroscopy of graphene and graphite: Disorder, electron-phonon coupling, doping and nonadiabatic eff ects. Sol. Stat. Commun. 2007; 143, 47-57.

18. Kumar R, Sakthivel R, Behura R, Mishra BK, Das D. Synthesis of magnetite nanoparticles from mineral waste. J Alloys Compd. 2015; 645: 398-404.

19. Puca M, Guerrero M, Tacuri E, López RG. Síntesis y caracterización de nanopartículas superparamagnéticas obtenidas por precipitación en microemulsión inversa para aplicaciones biomédica. Rev Soc Quím Perú. 2013; 79 (2): 99-106

20. Peña G, Rivera PA, González CH, Parra CA, Garzón AO, Landínez DA, et al. Efecto de la concentración de magnetita en la estructura, propiedades eléctricas y magnéticas de un material compuesto a base de resina de poliéster. Tecnologías. 2018; 41 (21): 13-27

21. Sun Q, Liu J, Cheng H, Mou Y, liu J, Peng Y, et al. Fabrication of 3D structures via direct ink wrinting of kaolin/grapheme oxide composite suspensions at ambient temperature. Ceram Int. 2019; 45(15): 18972-18979. 